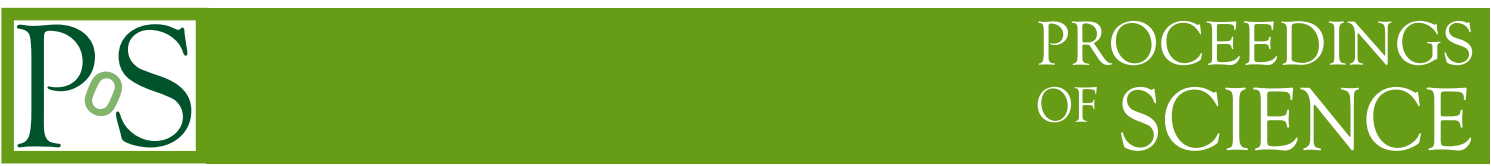

\title{
Implications of sgoldstino-Higgs mixing
}

\section{K. O. Astapov}

Institute for Nuclear Research of the Russian Academy of Sciences, 60th October Anniversary prospect 7a, Moscow 117312, Russia,

Faculty of Physics of Moscow State University,

Vorobjevy Gory, 119991, Moscow, Russia

E-mail: asapovk@gmail.com

\section{S. V. Demidov*}

Institute for Nuclear Research of the Russian Academy of Sciences, 60th October Anniversary prospect 7a, Moscow 117312, Russia

Faculty of Physics of Moscow State University,

Vorobjevy Gory, 119991, Moscow, Russia

E-mail: demidov@ms2.inr.ac.ru

We consider an supersymmetric extension of the Standard Model with low scale supersymmetry breaking. Besides usual superpartners it contains additional chiral goldstino supermultiplet whose scalar components - sgoldstinos, can mix with Higgs sector of the model. We show that this mixing can have considerable impact on phenomenology of the lightest Higgs boson and sgoldstino.

The XXI International Workshop High Energy Physics and Quantum Field Theory,

June 23 - June 30, 2013

Saint Petersburg Area, Russia

\footnotetext{
*Speaker.
} 


\section{Introduction}

Supersymmetry is still remains one of the most interesting extension of the Standard Model. The discovery of a light Higgs-like scalar resonance [1] with mass of order of $126 \mathrm{GeV}$ on the one hand supports idea of low energy supersymmetry. On the other hand to obtain such value of the Higgs boson mass requires a considerable degree of fine-tuning. It it well known that the mass of the lightest Higgs in the Minimal Supersymmetric Standard Model (MSSM) at tree level is bounded by Z-boson mass and it receives a large quantum correction (see Ref. [2] for our notations) from interactions with stop-quarks

$$
m_{h}^{2}=m_{Z}^{2} \cos ^{2} 2 \beta+\frac{3 m_{t}^{4}}{2 \pi^{2} v^{2}}\left[\log \left(\frac{m_{\tilde{t}}^{2}}{m_{t}^{2}}\right)+\frac{X_{t}^{2}}{m_{\tilde{t}}^{2}}\left(1-\frac{X_{t}^{2}}{12 m_{\tilde{t}}^{2}}\right)\right]
$$

where $X_{t}=A_{t}-\frac{\mu}{\tan \beta}$. Observed value of the Higgs mass implies either heavy stop contribution or maximal mixing in stop sector in the decoupling limit of the MSSM. This results in problems with naturalness criteria.

If supersymmetry is indeed relevant to our Nature it should be spontaneously broken in some hidden sector. According to supersymmetric analog of the Goldstone theorem [3] there should exist a massless fermionic degree of freedom, goldstino. After taking into account gravity goldstino becomes longitudinal component of gravitino with a mass related to the scale of supersymmetry breaking $F$ as follows $m_{3 / 2}=F /\left(\sqrt{3} M_{P l}\right)$. In the simplest case the goldstino is fermionic component of a chiral supermultiplet and interactions of this supermultiplet with other MSSM fields are suppressed by SUSY breaking scale $\sqrt{F}$. In most of the phenomenological applications it is typically assumed that this scale is large as compared to the electroweak scale. For instance, for gravity mediated SUSY breaking scenarios with soft parameters of order of TeV-scale this implies $\sqrt{F} \gtrsim 10^{11} \mathrm{GeV}$. For gauge mediation SUSY breaking scale can be considerably lower, but still its value is limited by $\sqrt{F} \gtrsim 50 \mathrm{TeV}$. At the same time given current results from LHC large scale of new physics implies high degree of fine-tuning in Higgs scalar potential. However, it is possible $[4,5,6]$ to have $\sqrt{F}$ not very far from electroweak scale, somewhere around several TeV. Recently, an interest to these models (so called low scale SUSY breaking scenario) has been renewed (see, e.g. [7, 8, 9] and references therein). In these models apart from usual supersymmetric spectrum goldstino and its superpartners - sgoldstinos, are presented in low energy theory at the electroweak scale. Phenomenology of the low scale supersymmetry and, in particular, sgoldstinos has been extensively studied in literature [10]. In this class of models gravitino is the lightest supersymmetric particle (LSP). It is phenomenologically possibles to have the masses of scalar and pseudoscalar sgoldstinos around electroweak scale. If these particles are light we have a possibility to probe the scale of supersymmetry breaking already at present-day experiments, in particular, at the LHC. In this talk we discuss mixing of sgoldstino with the Higgs sector of MSSM concentrating on the most interesting case of mixing with the lightest Higgs boson.

\section{A model}

Here we describe a minimal set of the interactions of goldstino supermultiplet with MSSM sector. To introduce them we follow spurion approach (see [11] and references therein). We denote 
goldstino chiral superfield as $\Phi=\phi+\sqrt{2} \theta \psi+F_{\phi}$, where $\phi=(S+i P) / \sqrt{2}$ represents sgoldstino degrees of freedom, $\psi$ is goldstino and $F_{\phi}$ is an auxiliary field. We introduce the interaction lagrangian given by the sum of the following contributions

$$
\begin{aligned}
\mathscr{L}_{\Phi-\text { Kähler }}= & -\int d^{2} \theta d^{2} \bar{\theta} \Phi^{\dagger} \Phi \cdot \sum_{\begin{array}{c}
\text { all matter } \\
\text { and Higs field }
\end{array}} \frac{m_{k}^{2}}{F^{2}} \Phi_{k}^{\dagger} e^{g_{1} V_{1}+g_{2} V_{2}+g_{3} V_{3}} \Phi_{k}, \\
\mathscr{L}_{\Phi-\text { gauge }}= & \frac{1}{2} \int d^{2} \theta \Phi \cdot \sum_{\begin{array}{c}
\text { all gauge } \\
\text { fields }
\end{array}} \frac{M_{\alpha}}{F} \operatorname{Tr} W^{\alpha} W^{\alpha}+h . c ., \\
\mathscr{L}_{\Phi-\text { superpotential }}= & \int d^{2} \theta \Phi \cdot \varepsilon_{i j}\left(-\frac{B}{F} H_{D}^{i} H_{U}^{j}+\frac{A_{a b}^{L}}{F} L_{a}^{j} E_{b}^{c} H_{D}^{i}+\frac{A_{a b}^{D}}{F} Q_{a}^{j} D_{b}^{c} H_{D}^{i}\right. \\
& \left.+\frac{A_{a b}^{U}}{F} Q_{a}^{i} U_{b}^{c} H_{U}^{j}\right)+ \text { h.c. }
\end{aligned}
$$

Note that the above lagrangian should be considered as an effective field theory which is valid at energies $E \lesssim \sqrt{F}$. To simplify matters we make the following assumptions. We will suppose that sgoldstino field does not acquire non-zero v.e.v. Further, we suppose that due to some interactions in the hidden sector the auxiliary field $F_{\phi}$ acquires non-zero vacuum expectation value $\left\langle F_{\phi}\right\rangle=F$ and SUSY becomes spontaneously broken and the above lagrangian reproduces soft terms of MSSM. Finally we introduce mass of sgoldstino fields $m_{S}$ and $m_{P}$ phenomenologically and assume that they are of order of electroweak scale.

Here we will be interesting in mixing of scalar sgoldstino $S$ with the lightest Higgs boson $h$ in the decoupling limit when $m_{h} \ll m_{A}, m_{H}$. We integrate out the auxiliary fields for Higgs doubles, goldstino superfield and vector superfields and obtain corrections to the lagrangian due to sgoldstino interactions. In this way we obtain a correction to the squared mass of the lightest Higgs boson

$$
m_{h^{0}}^{2}=m_{Z}^{2} \cos ^{2} 2 \beta+\frac{v^{2}}{F^{2}}\left(B \sin ^{2} 2 \beta-2 \mu^{2}\right)^{2}
$$

where $v$ is v.e.v. of the Higgs field. The appearance of this correction to the mass of the lightest Higgs boson has been observed in Ref. [7]. The mixing between the lightest Higgs boson $h$ and the scalar sgoldstino $S$ in the lowest order in $1 / F$ is given by $\mathscr{L}_{m i x}=\frac{X}{F} h \cdot S$ with

$$
X=2 \mu^{3} v \sin 2 \beta+\frac{1}{2} v^{3}\left(g_{1}^{2} M_{1}+g_{2}^{2} M_{2}\right) \cos ^{2} 2 \beta,
$$

In the case when sgoldstino mass parameter is much larger then $m_{h}$ the corrections to the masses of the scalar particles due to this mixing are suppressed. In the opposite situation, i.e. when mass parameter of sgoldstino is somewhat smaller than $m_{h}$, the mixing with sgoldstino can give rise an additional positive contribution to the lightest Higgs boson mass.

\section{The consequences of sgoldstino-Higgs mixing}

Here we discuss the phenomenological consequences of possible mixing of sgoldstino with the lightest Higgs boson assuming the decoupling limit. First of all let us discuss the changes of the branching ratios for the scalar sgoldstino [10] due to the mixing. Typically, the dominating 
decay channels are the decays into massless gauge bosons rather then to fermions like in the case of the SM Higgs boson. This is related to the fact that for the Higgs boson the couplings to the gluons and gammas arise only at one loop level as compared to the couplings to massive particles, while for sgoldstinos all couplings exist already at tree level. In the Fig. 1 we show how the picture of sgoldstino decays changes depending on mixing angle. We see that even small value of mixing angle can considerably change the hierarchy between possible decay channels and already at mixing angle of 0.4 the picture becomes very similar to the case of the Higgs.
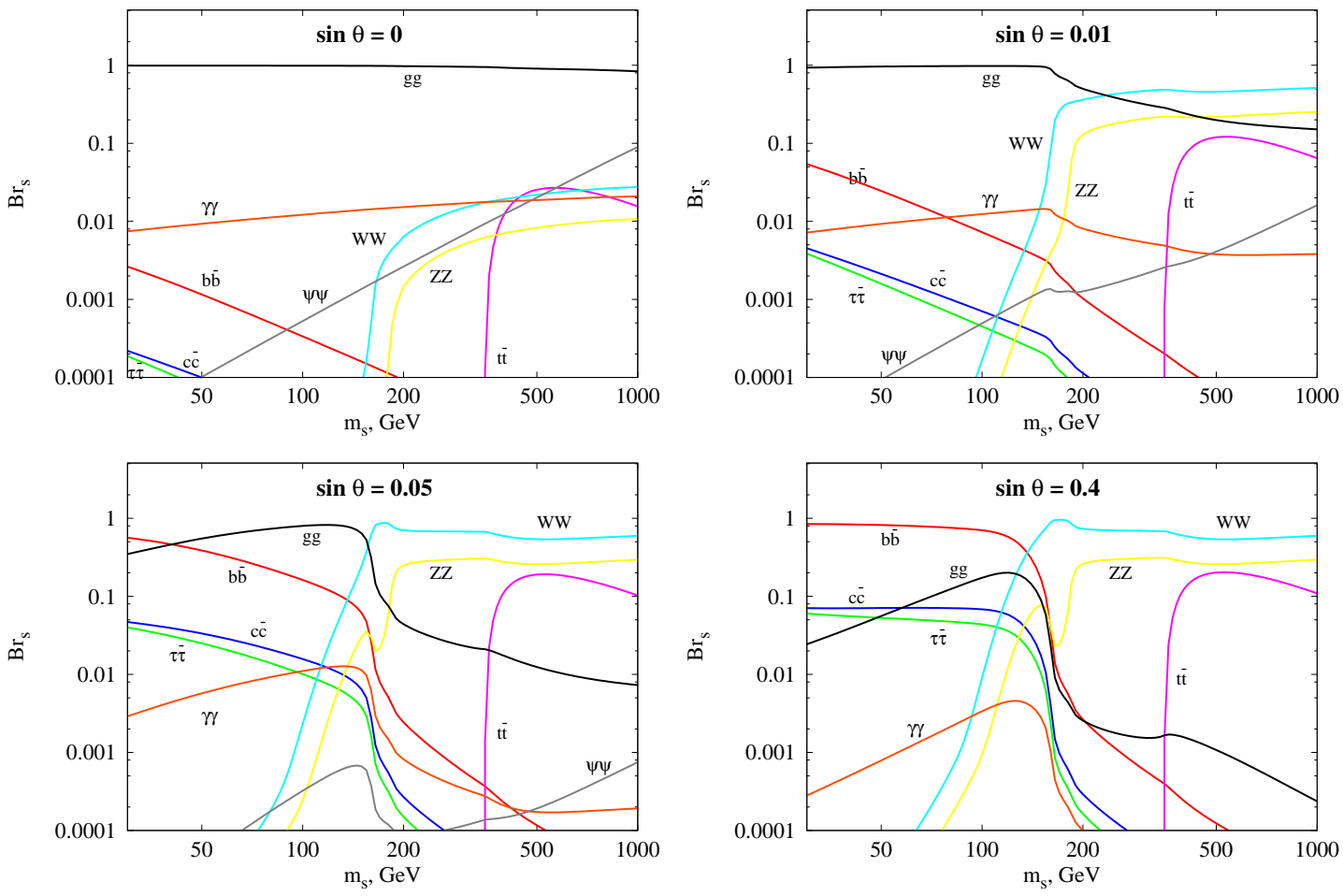

Figure 1: Modification of sgoldstino branching ratios at different values of mixing angle: $\sin \theta=$ $0.0,0.01,0.05$ and 0.4. We take the following values for MSSM soft parameters: $\sqrt{F}=10 \mathrm{TeV}, M_{1}=$ $400 \mathrm{GeV}, M_{2}=800 \mathrm{GeV}, M_{3}=-1200 \mathrm{GeV}, A^{U, D, E}=700 \mathrm{GeV}$ and $A_{a b}^{U, D, E}=Y_{a b}^{U, D, E} A^{U, D, E}$ where $Y_{a b}^{U, D, E}$ are Yukawa couplings.

To explore whether this scenario is indeed possible in the low scale MSSM we make a scan over some MSSM parameters space inspired by naturalness. We use a package NMSSMTools [12] in the MSSM regime and vary MSSM parameters with the followings intervals: $1.5<\tan \beta<50.0$, $100 \mathrm{GeV}<\mu<1500 \mathrm{GeV}, 100 \mathrm{GeV}<M_{1}<500 \mathrm{GeV}, 200 \mathrm{GeV}<M_{2}<500 \mathrm{GeV}, 1.5 \mathrm{TeV}<M_{3}<$ $2.0 \mathrm{TeV},\left|A^{U, D, E}\right|<1.5 \mathrm{TeV}, 700 \mathrm{GeV}<m_{Q_{3}}<1.0 \mathrm{TeV}, 1.0 \mathrm{TeV}<m_{U_{3}}, m_{D_{3}}<1.3 \mathrm{TeV}$ while soft masses of the first and second generation squarks and sleptons are taken to be sufficiently large. We fix $\sqrt{F}=10 \mathrm{TeV}$. Also we scan uniformly over sgoldstino mass parameter $m_{S}$ in the following range $85 \mathrm{GeV}<m_{S}<115 \mathrm{GeV}$. We imposed the main experimental constraints including constraints from LEP and generic constraints on superpartner masses from LHC experiments. For each model we calculate the signal strengths $R_{\gamma \gamma}^{g g}, R_{Z Z}^{g g}, R_{\tau^{+} \tau^{-}}^{g g}$ and $R_{b \bar{b}}^{V B F}$, i.e. the ratios of signal cross sections for the new Higgs to the cross section in the SM case in the corresponding channel.

Some results of this scanning are presented in Fig. 2. In this plot we show the mass of the 

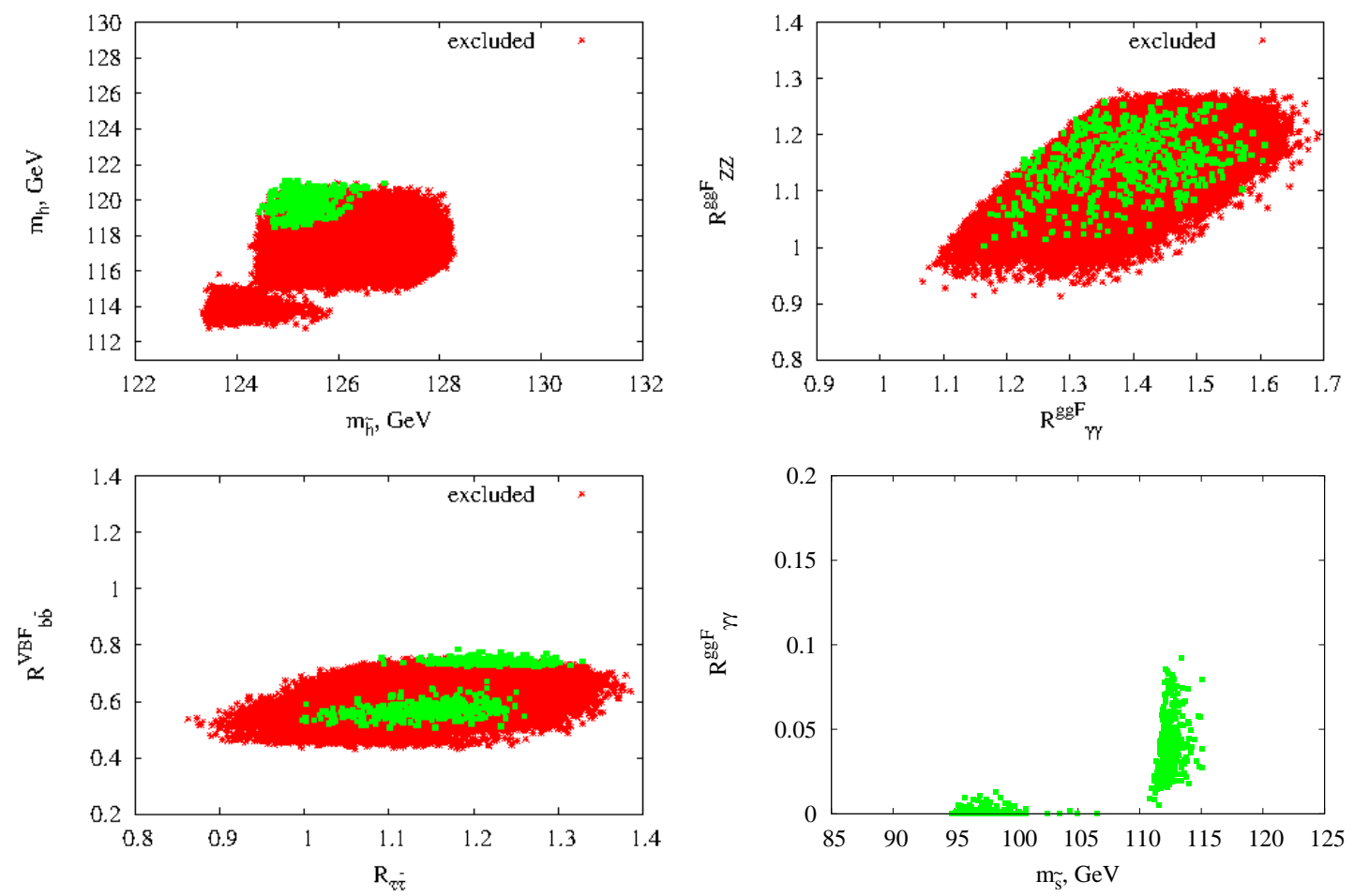

Figure 2: Mass of the lightest Higgs boson before $m_{h}$ and after $m_{\tilde{h}}$ mixing (upper, left). Signal strengths for for the Higgs-like resonance in $\gamma \gamma$ and $Z Z$ channels in gluon-gluon fusion (upper, right). Signal strengths for the Higgs-like resonance in $b \bar{b}$ in VBF and $\tau \tau$ in gluon-gluon fusion (lower, left). Signal strength for sgoldstino-like state in $\gamma \gamma$ channel (lower, right).

lightest Higgs before and after mixing (upper, left). It is clearly seen that before mixing Higgs mass does not become larger $122 \mathrm{GeV}$ while the mixing can bring its value inside the observed range $124 \mathrm{GeV}<m_{\tilde{h}}<127 \mathrm{GeV}$. Here by red points we show the models which are already excluded either by experimental constraints (in particular, by LEP constraints). On the upper, right plot we show the LHC signal strength relative to the SM Higgs of the same mass for $\gamma \gamma$ and $Z Z$ channels. One can see that the signal strength can differ from unity for some models which could be a signature of an admixture of some scalar particle to the Higgs boson. Also the model predicts some decrease in the signal strength for $b \bar{b}$ in vector boson fusion production channel. At the same time now another sgoldstino-like scalar is present in the spectrum and on the right lower plot we present corresponding signal strength for the most promising $\gamma \gamma$ channel in gluon-gluon fusion. One can see that it can reach values of about 0.2 . We note, that only limited parameter space is phenomenologically acceptable here because of exclusion from LEP data. More thorough analysis of the phenomenology of such a scenario will be presented elsewhere [13].

\section{Conclusions}

In models with low scale supersymmetry mixing of sgoldstinos with Higgs sector can have considerable impact on phenomenology. In particular, mixing with sgoldstino can give additional positive contribution to the Higgs mass which can push its value to the observed $125 \mathrm{GeV}$ with- 
out considerable violation of naturalness criteria. One of the consequence of this scenario is an additional scalar which can be probed at the experiments at LHC.

Acknowledgements We thank D. Gorbunov for valuable discussions. The work was supported in part by the grants of the President of the Russian Federation NS-5590.2012.2, MK-2757.2012.2, by Russian Foundation for Basic Research grants 11-02-01528-a (S.D.), 13-02-01127 (S.D.) and 12-02-31726-mol-a and by the Ministry of Science and Education under contract No. 8412. The numerical part of the work was performed on Calculational Cluster of the Theory Division of INR RAS.

\section{References}

[1] G. Aad et al. [ATLAS Collaboration], Phys. Lett. B 716 (2012) 1 [arXiv:1207.7214 [hep-ex]], S. Chatrchyan et al. [CMS Collaboration], Phys. Lett. B 716 (2012) 30 [arXiv:1207.7235 [hep-ex]].

[2] S. P. Martin, In *Kane, G.L. (ed.): Perspectives on supersymmetry II* 1-153 [hep-ph/9709356].

[3] D. V. Volkov and V. P. Akulov, JETP Lett. 16 (1972) 438 [Pisma Zh. Eksp. Teor. Fiz. 16 (1972) 621], D. V. Volkov and V. P. Akulov, Phys. Lett. B 46 (1973) 109.

[4] G. F. Giudice and R. Rattazzi, Phys. Rept. 322 (1999) 419 [hep-ph/9801271], S. L. Dubovsky, D. S. Gorbunov and S. V. Troitsky, Phys. Usp. 42 (1999) 623 [Usp. Fiz. Nauk 169 (1999) 705] [hep-ph/9905466].

[5] J. R. Ellis, K. Enqvist and D. V. Nanopoulos, Phys. Lett. B 147 (1984) 99, J. R. Ellis, K. Enqvist and D. V. Nanopoulos, Phys. Lett. B 151 (1985) 357.

[6] A. Brignole, F. Feruglio and F. Zwirner, Nucl. Phys. B 501 (1997) 332 [hep-ph/9703286], A. Brignole, J. A. Casas, J. R. Espinosa and I. Navarro, Nucl. Phys. B 666 (2003) 105 [hep-ph/0301121].

[7] C. Petersson and A. Romagnoni, JHEP 1202 (2012) 142 [arXiv:1111.3368 [hep-ph]].

[8] B. Bellazzini, C. Petersson and R. Torre, Phys. Rev. D 86 (2012) 033016 [arXiv:1207.0803 [hep-ph]].

[9] E. Dudas, C. Petersson and P. Tziveloglou, Nucl. Phys. B 870 (2013) 353 [arXiv:1211.5609 [hep-ph]].

[10] E. Perazzi, G. Ridolfi and F. Zwirner, Nucl. Phys. B 574 (2000) 3 [hep-ph/0001025], E. Perazzi, G. Ridolfi and F. Zwirner, Nucl. Phys. B 590 (2000) 287 [hep-ph/0005076], D. S. Gorbunov, Nucl. Phys. B 602 (2001) 213 [hep-ph/0007325], D. S. Gorbunov and V. A. Rubakov, Phys. Rev. D 64 (2001) 054008 [hep-ph/0012033], D. Gorbunov, V. Ilyin and B. Mele, Phys. Lett. B 502 (2001) 181 [hep-ph/0012150], D. S. Gorbunov and N. V. Krasnikov, JHEP 0207 (2002) 043 [hep-ph/0203078], S. V. Demidov and D. S. Gorbunov, Phys. Atom. Nucl. 69 (2006) 712 [hep-ph/0405213], D. S. Gorbunov and V. A. Rubakov, Phys. Rev. D 73 (2006) 035002 [hep-ph/0509147], S. V. Demidov and D. S. Gorbunov, JETP Lett. 84 (2007) 479 [hep-ph/0610066], S. V. Demidov and D. S. Gorbunov, Phys. Rev. D 85 (2012) 077701 [arXiv:1112.5230 [hep-ph]],

[11] D. S. Gorbunov and A. V. Semenov, hep-ph/0111291.

[12] U. Ellwanger, J. F. Gunion and C. Hugonie, JHEP 0502 (2005) 066 [hep-ph/0406215], U. Ellwanger and C. Hugonie, Comput. Phys. Commun. 175 (2006) 290 [hep-ph/0508022], G. Belanger, F. Boudjema, C. Hugonie, A. Pukhov and A. Semenov, JCAP 0509 (2005) 001 [hep-ph/0505142].

[13] K. O. Astapov, S. V. Demidov, in preparation. 